\title{
Estudo clínico e epidemiológico das anemias em pacientes hospitalizados
}

\author{
Clinical and epidemiological study of anemia in hospitalized patients
}

\author{
Mariana Franco de Oliveira ${ }^{1}$, Pedro Castela Malozze $^{1}$ Marília Franco de Oliveira $^{1}$, \\ Nathalya Fernanda Rossi ${ }^{1}$, Juliana Rinaldis da Silva ${ }^{1}$, Nathalia Cavalcante Navarro ${ }^{1}$, \\ Michael da Cruz Ferrari ${ }^{1}$, Renata de Oliveira Costa ${ }^{2}$, Daniel D’Almeida Preto ${ }^{3}$
}

Oliveira MF, Malozze PC, Oliveira MF, Rossi NF, Silva JR, Navarro NC, Ferrari MC, Costa RO, Preto DA. Estudo clínico e epidemiológico das anemias em pacientes hospitalizados / Clinical and epidemiological study of anemia in hospitalized patients. Rev Med (São Paulo). 2019 jan.-fev.;98(1):23-9.

RESUMO: A anemia é uma condição clínica frequente que pode ocorrer especialmente em pacientes hospitalizados em razão de múltiplas comorbidades, como neoplasias, insuficiência renal e infecções. A presença de estudos que avaliem o real impacto da anemia no sistema de saúde e desfecho hospitalar são escassos, portanto o presente estudo tem por objetivo identificar as etiologias das anemias e avaliar o impacto no tempo de internação e mortalidade de pacientes hospitalizados. Esse estudo prospectivo transversal avaliou 268 pacientes, acima de 16 anos, admitidos para hospitalização na clínica médica no período de 01 de agosto a 31 de outubro de 2016. Os pacientes foram acompanhados até o desfecho final de alta hospitalar ou óbito. No que concerne aos resultados, $66,3 \%$ dos pacientes apresentaram anemia, com idade mediana de 56,6 anos e predomínio no sexo masculino. A maior prevalência foi de anemia normocítica normocrômica, cuja etiologia principal observada foi anemia de doença crônica (99 pacientes; 80,48\%), também conhecida como anemia da inflamação. Em relação ao grau da anemia e a mortalidade, observou-se que esta foi superior nos pacientes que apresentaram anemia moderada/severa $(36,4 \%)$ em comparação com aqueles diagnosticados com anemia leve (18,2\%). Esse estudo evidenciou uma maior prevalência da anemia de doença crônica/anemia da inflamação nos pacientes hospitalizados, o que contribuiu para um maior tempo de internação e pior desfecho. Pelo fato da anemia ser um sinal e não um diagnóstico etiológico, estabelecer sua causa é mandatório, pois a implementação de uma terapia eficaz melhora a qualidade de vida do paciente anêmico e pode reduzir o impacto na mortalidade daqueles internados.

Descritores: Anemia; Pacientes internados; Contagem de células sanguíneas; Anemia ferropriva.

\begin{abstract}
Anemia is a frequent clinic condition that occurs especially in hospitalized patients due to multiple comorbidities, as neoplasms, renal insufficiency and infections. The present study aimed to identify the anemia's etiology and evaluate the impact on the period of hospitalization and mortality, due to lack of studies that assess the real impact of anemia in the health system and hospital outcomes. Participants in this prospective study included 268 patients over 16 years old, admitted for hospitalization at medical clinic in the period of August $1^{\text {st }}$ of 2016 to October $31^{\text {st }}$ of 2016 . Those patients were followed to hospital discharge or death. Regarding the results, $66.3 \%$ presented anemia, with median age of 56.6 years and men having a lower mean hemoglobin concentration than women. The most prevalent was normocytic and normochromic anemia, and main etiology was chronic disease (99 patients; $80.48 \%$ ), also known as anemia of inflammation. Regarding the severity of anemia and mortality it was observed higher rates of death in patients presenting moderate/severe anemia (36.4\%) compared to those with mild anemia $(18.2 \%)$. The study reveals higher prevalence of anemia of chronic disease/anemia of inflammation in hospitalized patients, which contributed to a longer hospital stay and worse outcomes. By the fact anemia is a clinical sign and not an etiologic diagnosis, it's mandatory to establish a cause in order to treat correctly through the implementation of an effective therapy to enhance quality of life of anemic patients and possible reducing mortality in those hospitalized.
\end{abstract}

Keywords: Anemia; Inpatients; Blood cell count; Anemia, irondeficiency.

Trabalho realizado no Centro Universitário Lusíada (Unilus) - Campus 2. Santos, SP, Brasil.

1. Graduanda(o) do Curso de Medicina do Centro Universitário Lusíada (Santos-SP). ORCID: Oliveira MF - https://orcid.org/0000-0002-75207124; Malozze PC - https://orcid.org/0000-0002-8694-9908; Oliveira MF - https://orcid.org/0000-0002-7907-9211; Rossi NF - https://orcid. org/0000-0001-8471-0883; Silva JR -https://orcid.org/0000-0001-9372-9818; Navarro NC - https://orcid.org/0000-0001-6985-2445; Ferrari MC - https://orcid.org/0000-0002-3333-7158. Email: mafrancooliveira@gmail.com; pedrocmalozze@gmail.com; marilia_fo@hotmail.com; rossinathalya@gmail.com; rinaldis.juliana@gmail.com; natlhalia_nav@hotmail.com; michferrarirt@hotmail.com.

2. Orientadora. Professora de Hematologia pelo Centro Universitário Lusíadas - Unilus, Faculdade de Ciências Médicas de Santos. https:// orcid.org/0000-0002-3345-3546. Email: renatadeoliveiracosta@uol.com.br.

3. Residência médica em Oncologia Clínica em andamento pelo Hospital de Câncer de Barretos (Fundação Pio XII). https://orcid.org/00000002-6561-7322. Email: ddpreto@gmail.com.

Endereço para correspondência: Mariana Franco de Oliveira. Av. Vicente de Carvalho, no 65 - Apto 52. Gonzaga, Santos, SP. CEP: $11045-$ 501. Email: mafrancooliveira@gmail.com. 


\section{INTRODUÇÃO} anemia é uma condição clínica freqüente
que pode ocorrer em diversas doenças, especialmente em pacientes hospitalizados em razão de múltiplas comorbidades, como neoplasias, insuficiência renal e infecções. A identificação da etiologia anêmica, dessa forma, merece atenção especial ${ }^{1,2}$.

Diferentes estudos mostraram a relação entre anemia e baixo estado cognitivo, piora da qualidade de vida e associação com a depressão ${ }^{3}$, bem como aumento do número de quedas da própria altura e falência cardíaca congestiva. A miríade de influências prognósticas que a anemia exerce, portanto, pode ser resumida como uma redução da sobrevida, aumento no número de hospitalizações e tempo de internação prolongado ${ }^{2,3}$.

Como fator agravante, a anemia continua sendo subdiagnosticada e negligenciada, proporcionando, desta forma, impacto sócio econômico negativo, considerando os altos custos de hospitalização ${ }^{4}$.

Segundo a Organização Mundial da Saúde (OMS), a anemia é definida pela redução dos níveis de hemoglobina (Hb) para valores inferiores a $12 \mathrm{~g} / \mathrm{dL}$ em mulheres não gestantes e $13 \mathrm{~g} / \mathrm{dL}$ em homens (OMS,1968). Apesar de universalmente aceito, esse critério da OMS é uma forma arbitrária de classificar um paciente como portador de anemia, pois desconsidera diferenças étnicas ${ }^{5}$.

Estudos que avaliem o real impacto da anemia no sistema de saúde e desfecho hospitalar são escassos e disparidades no valor de corte de hemoglobina para definir a anemia, bem como heterogeneidades nos estudos abordando a sua etiologia impedem a formação de um panorama brasileiro sobre o tema. Assim, buscando compreendê-las sob o perfil epidemiológico em pacientes hospitalizados, este estudo tem como objetivo identificar suas etiologias, avaliando o impacto no tempo de internação e na mortalidade.

\section{MATERIAS E MÉTODOS}

Realizou-se um estudo longitudinal prospectivo em 268 pacientes admitidos para hospitalização na clínica médica do Hospital Guilherme Álvaro, de 01 de agosto e 31 de outubro de 2016.

Foram incluídos pacientes com faixa etária acima de 16 anos internados consecutivamente no setor, e que apresentavam anemia segundo os critérios de elegibilidade da (OMS): hemoglobina $(\mathrm{Hb})$ inferior à $12 \mathrm{mg} / \mathrm{dl}$ para mulheres e $13 \mathrm{mg} / \mathrm{dl}$ para homens. Uma subclassificação proposta pela mesma entidade define anemia grave como aquela com valores de $\mathrm{Hb}$ inferiores a $8 \mathrm{~g} / \mathrm{dl}$, moderada de $8 \mathrm{~g} / \mathrm{dl}$ a $10,9 \mathrm{~g} / \mathrm{dl}$ e anemia leve subdividida em sexo, sendo o intervalo de $11 \mathrm{~g} / \mathrm{dl}$ a $11,9 \mathrm{~g} / \mathrm{dl}$ para mulheres e $11 \mathrm{~g} / \mathrm{dl}$ a $12,9 \mathrm{~g} / \mathrm{dl}$ para homens ${ }^{6}$.
Foram excluídas gestantes, pacientes que haviam recebido hemocomponentes nos últimos 60 dias, pacientes sem hemograma, pacientes com desfecho antes do diagnóstico do tipo de anemia ou que se recusaram a participar.

Os dados dos pacientes foram obtidos em formulário próprio elaborado para o estudo incluindo informações sócio demográficas, anamnese e exame físico e o hemograma obtido no equipamento Cell-Dyn Ruby em amostra de sangue total coletado em tubo contendo ácido etilenodiamino tetra-acético (EDTA). O formulário era composto por: sexo, idade, data de internação, desfecho hospitalar, tempo de internação, motivo da internação, diagnóstico clínico, classificação morfológica da anemia, grau de severidade da anemia definido pelo valor da hemoglobina, dosagem sérica da série branca e plaquetas. As informações foram revisadas com a hematologista responsável e foram realizados exames complementares para o diagnóstico morfológico e etiopatogênico da anemia. A complementação diagnóstica da anemia baseou-se na contagem de reticulócitos, dosagem de ferritina, ferro sérico, saturação de transferrina e mielograma quando pertinente. Os pacientes foram acompanhados até o desfecho final de alta hospitalar ou óbito.

A análise descritiva dos pacientes foi realizada por meio de mediana, média e desvio padrão para variáveis quantitativas e frequência relativa e absoluta para variáveis qualitativas. Para verificar as diferenças das variáveis independentes frente à gravidade da anemia utilizou-se teste Qui-quadrado. Na presença de caselas vazias, optou-se pelo teste exato de Fisher. Para verificar a diferença entre variáveis quantitativas, primeiramente realizou-se teste de tendência à normalidade (Teste de Shapiro). Para variáveis paramétricas utilizou-se teste t de Student para verificar diferença de médias, para variáveis não paramétricas, teste de Mann-Whitney e apresentação de resultados por meio gráfico, utilizando Boxplot.

Considerou-se nível de significância de 5\% para o presente estudo. Todas as análises foram realizadas utilizando-se o software Stata, versão 13.1.

Esse estudo foi aprovado pelo Comitê de Ética e Pesquisa do Hospital Guilherme Alvaro, sob n ${ }^{\circ}$ CAAE: 57862316.4.0000.5448.

\section{RESULTADOS}

De agosto a outubro de 2016 foram internados na Enfermaria de Clínica Médica do Hospital Guilherme Álvaro 268 pacientes. Destes, 66,3\% apresentaram anemia (Figura 1), com idade mediana de 56,6 anos e predomínio no sexo masculino (Tabela 1 ).

Quanto a classificação morfológica observou-se predomínio de anemia normocítica normocrômica (Figuras 2 e 3 ). 


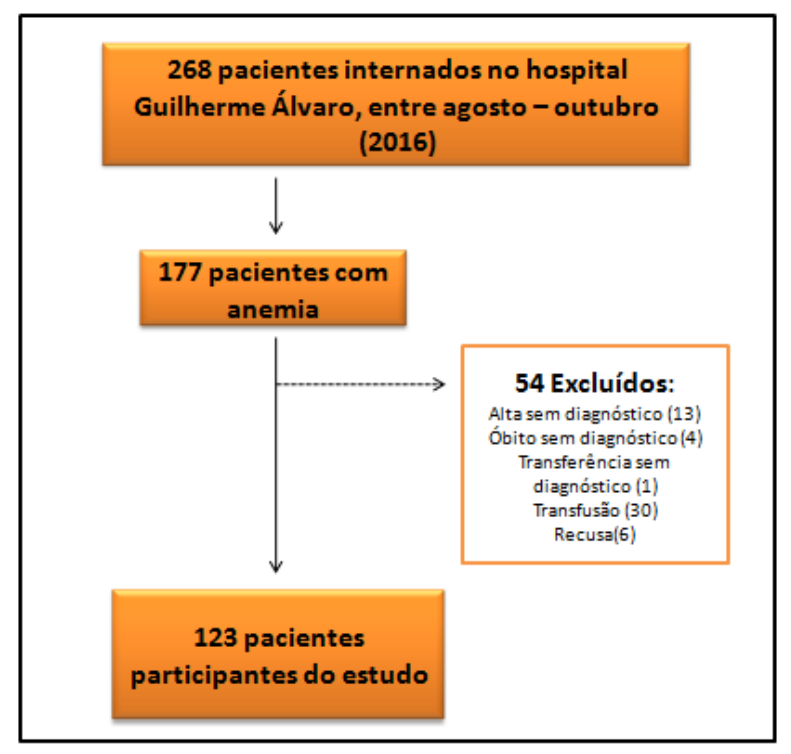

Figura 1. Fluxograma de amostra do estudo

Tabela 1. Descritiva das características demográficas de todos os pacientes internados e dos pacientes com anemia diagnosticada no período do estudo (ago.-out.). Santos, 2016

\begin{tabular}{lcc}
\hline Total & $\begin{array}{c}\text { Pacientes } \\
\text { Internados }\end{array}$ & $\begin{array}{c}\text { Total de pacientes } \\
\text { com Anemia }\end{array}$ \\
Sexo & $268(100)$ & $177(66.3)$ \\
Masculino & $140(52.0)$ & $99(55.9)$ \\
Feminino & $128(48.0)$ & $78(44.1)$ \\
Idade* & $56.3(18.6)$ & $56.6(18.0)$ \\
Idade & & \\
(categorizada) & $135(50.4)$ & $90(50.9)$ \\
18 - 59 anos & $133(49.6)$ & $87(49.1)$ \\
60 anos e mais & & \\
Gravidade da anemia $* *$ & \\
Leve & & $33(26.6)$ \\
Moderada & & $71(57.3)$ \\
Grave & & $20(16.1)$ \\
\hline
\end{tabular}

* Média e desvio padrão. **Gravidade referente aos pacientes incluídos no estudo (n=123). Hospital Guilherme Àlvaro, Santos, 2016

A doença de base que motivou a internação, o tempo de internação, classificação etiopatogênica da anemia e desfechos não apresentaram significância estatística que os relacione com a gravidade da anemia (Tabela 2).

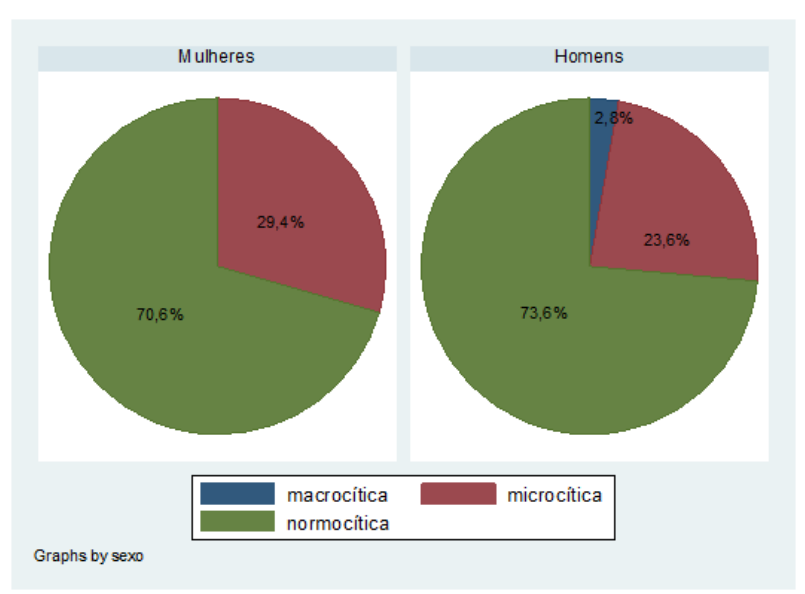

Figura 2. Classificação morfológica segundo sexo. Santos, 2016

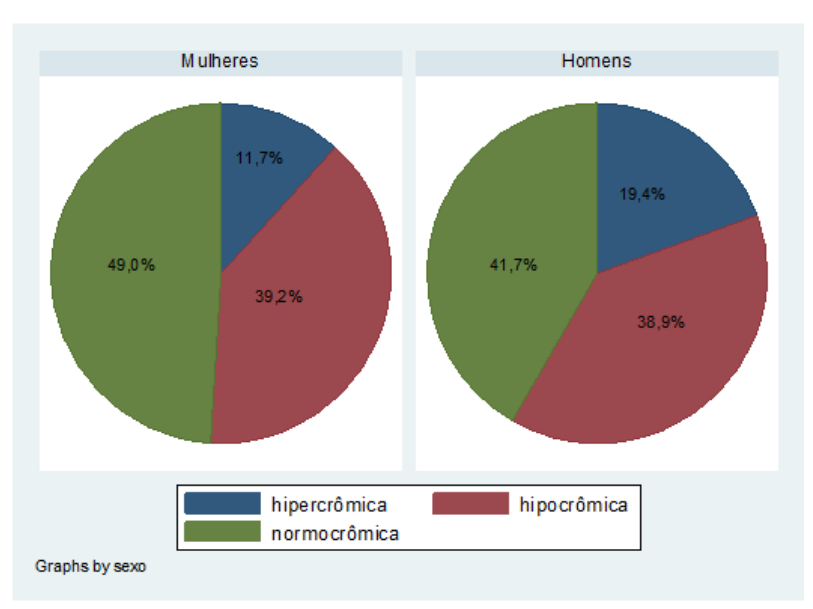

Figura 3. Classificação HCM segundo sexo. Santos, 2016

A doença de base que motivou a internação, o tempo de internação, classificação etiopatogênica da anemia e desfechos não apresentaram significância estatística que os relacione com a gravidade da anemia (Tabela 2).

Ao correlacionarmos o grau de anemia e sua distribuição por idade verificou-se o predomínio em pacientes acima de 60 anos, com a seguinte distribuição: leve $25 \%(n=16)$; moderada $62,5 \%(n=40)$; grave $12,5 \%(n=8)$. Já nos pacientes abaixo de 60 anos a distribuição encontrada foi: leve 28,8\% $(n=17)$; moderada $52,5 \%(\mathrm{n}=31)$; grave $18,6 \%(\mathrm{n}=11)$.

Ao hemograma, verificou-se que os pacientes com anemia grave apresentavam maior prevalência de alterações de leucócitos e plaquetas, com significância estatística, $\mathrm{p}=\mathrm{de}$ 0,044 e 0,043 , respectivamente (Tabela 3). 
Oliveira MF, et al. Estudo clínico e epidemiológico das anemias em pacientes hospitalizados.

Tabela 2. Descrição das características de saúde e desfecho hospitalar de pacientes diagnosticados com anemia, segundo gravidade da anemia. Santos, 2016

\begin{tabular}{|c|c|c|c|}
\hline & Leve & $\begin{array}{l}\text { Moderada/ } \\
\text { Grave }\end{array}$ & p valor \\
\hline Total & $33(26.83)$ & 91(73.17) & \\
\hline \multicolumn{4}{|l|}{ Motivo de internação } \\
\hline Diagnóstico & $23(69.7)$ & $46(50.6)$ & 0.058 \\
\hline Tratamento & $10(30.3)$ & $45(49.4)$ & \\
\hline \multicolumn{4}{|l|}{$\begin{array}{l}\text { Etiopatogênsese da } \\
\text { anemia }^{1}\end{array}$} \\
\hline Aplásica & $0(0)$ & $1(1.1)$ & 0.430 \\
\hline Carencial & $3(9.1)$ & $1(1.1)$ & \\
\hline $\begin{array}{l}\text { Relacionada à doença } \\
\text { crônica }\end{array}$ & $26(78.8)$ & 73(81.1) & \\
\hline Infiltração medular & 1(3) & $3(3.3)$ & \\
\hline Hemolítica & $0(0)$ & $3(3.3)$ & \\
\hline Multifatorial & $2(6.1)$ & $6(6.7)$ & \\
\hline Desconhecida & 1(3) & $3(3.3)$ & \\
\hline \multicolumn{4}{|l|}{ Número de causas } \\
\hline 0 & $1(3.0)$ & $3(3.4)$ & 0.699 \\
\hline 1 & $26(78.8)$ & $69(77.5)$ & \\
\hline 2 & $4(12.2)$ & $15(16.9)$ & \\
\hline 3 & $2(6.1)$ & $2(2.2)$ & \\
\hline \multicolumn{4}{|l|}{ Desfecho * } \\
\hline Alta & $24(74.7)$ & $52(59.1)$ & 0.129 \\
\hline Óbito & $6(18.2)$ & $32(36.4)$ & \\
\hline Internado & $3(9.1)$ & $4(4.5)$ & \\
\hline $\begin{array}{l}\text { Tempo médio de } \\
\text { internação** }\end{array}$ & 14.9 & 15.8 & 0.721 \\
\hline
\end{tabular}

1 - Procedido Teste exato de Fisher. *Desfecho relacionado à transferência (1) e evasão (1) ocorreram apenas para o grupo com anemia moderada ou grave. **Teste t de Student para diferença de médias.

Houve diferença no valor do RDW quanto à gravidade da anemia. Pacientes com anemia mais graves apresentaram maiores índices de anisocitose, com tendência de diferença mediana significativa em relação aos pacientes com anemia leve.

Por meio de um modelo de regressão logística univariado observou-se que pacientes com leucocitose clonal apresentaram probabilidade de 4,05 vezes maior de ter anemia moderada ou grave em comparação aos pacientes sem leucocitose clonal (p valor 0,020) (Tabela 4). Esses pacientes também tiveram maior evolução para o desfecho óbito $(p=0,008)$ em relação às demais alterações leucocitárias (Tabela 5).
Tabela 3. Descrição da análise sanguínea de pacientes diagnosticados com anemia, segundo gravidade da anemia. Santos, 2016

\begin{tabular}{|c|c|c|c|}
\hline & Leve & $\begin{array}{l}\text { Moderada/ } \\
\text { grave }\end{array}$ & $\begin{array}{c}\mathbf{p} \\
\text { valor }\end{array}$ \\
\hline \multicolumn{4}{|l|}{ Classificação VCM } \\
\hline Normal & $23(69.7)$ & $66(73.3)$ & 0.578 \\
\hline Microcítica & $10(30.3)$ & $22(24.4)$ & \\
\hline Macrocítica & $0(0)$ & $2(2.2)$ & \\
\hline \multicolumn{4}{|l|}{ Classificação HCM } \\
\hline Nomocrômica & $14(42.4)$ & $41(45.6)$ & 0.334 \\
\hline Hipocrômica & $11(33.3)$ & $37(41.1)$ & \\
\hline Hipercrômica & $8(24.3)$ & $12(13.3)$ & \\
\hline \multicolumn{4}{|l|}{ Classificação RDW } \\
\hline Normal & $6(18.2)$ & $32(36.4)$ & 0.055 \\
\hline Aumentado & $27(81.8)$ & $56(63.4)$ & \\
\hline \multicolumn{4}{|l|}{ Classificação reticulócitos } \\
\hline Hipoproliferativo & $24(96.0)$ & $68(90.7)$ & 0.395 \\
\hline Não hipoproliferativo & $1(4.0)$ & $7(9.3)$ & \\
\hline \multicolumn{4}{|l|}{ Classificação leucócitos } \\
\hline Normal & $21(63.6)$ & $35(38.9)$ & 0.044 \\
\hline Leucocitose clonal & $4(12.1)$ & $27(30.0)$ & \\
\hline Leucocitose reacional & $6(18.2)$ & $14(15.5)$ & \\
\hline Leucopenia & $2(6.1)$ & $14(15.6)$ & \\
\hline \multicolumn{4}{|l|}{ Classificação neutrófilos } \\
\hline Normal & $18(56.3)$ & $35(40.2)$ & 0.27 \\
\hline Neutrofilia & $13(40.6)$ & $46(52.9)$ & \\
\hline Neutropenia & $1(3.1)$ & $6(6.9)$ & \\
\hline \multicolumn{4}{|l|}{ Classificação linfócitos } \\
\hline Normal & $20(62.5)$ & $50(57.5)$ & 0.452 \\
\hline Linfocitose & $1(3.1)$ & $9(10.3)$ & \\
\hline Linfopenia & 11(34.4) & $28(32.2)$ & \\
\hline \multicolumn{4}{|l|}{ Classificação plaquetas $^{1}$} \\
\hline Normal & $27(81.8)$ & $50(54.6)$ & 0.043 \\
\hline Plaquetose clonal & $2(6.0)$ & $16(17.8)$ & \\
\hline Plaquetose reacional & $0(0)$ & $7(7.8)$ & \\
\hline Plaquetopenia & $4(12.1)$ & 17(18.9) & \\
\hline
\end{tabular}

Classificação de contagem celular periférica ${ }^{1}$

\begin{tabular}{lccc} 
Monocitopenia & $27(84.4)$ & $71(81.6)$ & 0.665 \\
Bicitopenia A+P** & $2(12.5)$ & $10(11.5)$ \\
Bicitopenia A+N** & $1(3.1)$ & $2(2.3)$ \\
Pancitopenia & $0(0)$ & $4(4.6)$ \\
\hline Diferença média avalia por teste t de Student. 1 - procedido Teste \\
to de Fisher. **A:anemia, P:plaquetopenia, N:neutropenia.
\end{tabular}
exato de Fisher. **A:anemia, P:plaquetopenia, N:neutropenia. 
Tabela 4. Modelo de regressão logística univariado para verificar a associação entre variáveis independentes e grau moderado ou grave de anemia. Santos, 2016

\begin{tabular}{lcc}
\hline & OR & p valor \\
\hline Motivo de internação & & \\
Diagnóstico & Ref & \\
Tratamento & 2.3 & 0.055 \\
Classificação RDW & & \\
Normal & Ref & \\
Aumentado & 0.39 & 0.06 \\
Classificação leucócitos & & \\
Normal & Ref & \\
Leucocitose clonal & 4.05 & 0.020 \\
Leucocitose reacional & 1.4 & 0.548 \\
Leucopenia & 4.5 & 0.061 \\
Classificação plaquetas & \\
Normal & & \\
Plaquetopenia & Ref & \\
Plaquetose clonal & 2.43 & 0.140 \\
Plaquetose reacional & 4.32 & 0.063
\end{tabular}

Tabela 5. Associação da condição leucocitária de paciente com anemia segundo desfecho óbito. Santos, 2016

\begin{tabular}{lll}
\hline Classificação leucócitos & Sobrevivente & Óbito \\
\hline Normal & $44(78.6)$ & $12(21.4)$ \\
Leucocitose clonal & $14(45.2)$ & $17(54.8)$ \\
Leucocitose reacional & $16(80.0)$ & $4(20.0)$ \\
Leucopenia & $11(68.8)$ & $5(31.2)$ \\
\hline *p valor 0,008 - Teste Qui-Quadrado &
\end{tabular}

\section{DISCUSSÃO}

Neste estudo, realizado em pacientes hospitalizados no serviço de clínica médica, a presença de anemia foi encontrada mais frequentemente no sexo masculino, preferencialmente em torno dos 56 anos. A principal etiopatogênese verificada foi anemia de doença crônica, também conhecida como anemia da inflamação. Já em relação à morfologia, a maior prevalência ocorreu entre as anemias normocrômicas e normocíticas, com destaque para os quadros clínicos de gravidade moderada, além disso a presença de anisocitose esteve mais relacionada a anemia moderada e grave.

A anemia possui caráter multifatorial, sofrendo influência de variáveis como idade, sexo e etnia. Somado a isso, os parâmetros utilizados para sua definição em diferentes estudos populacionais geralmente são bastante variáveis dado a ausência de consenso dos valores de hemoglobina adotados, fazendo com a prevalência na população seja heterogênea ${ }^{7-10}$.

Nos países em desenvolvimento como o Brasil, existe uma elevada prevalência de anemia na população em geral, uma vez adotados os critérios da OMS. Nesse grupo destaca-se a anemia por deficiência de ferro. Ao se restringir a análise epidemiológica para a população hospitalizada, a anemia de doença crônica ocupa a primeira posição, superando a ferropriva. Estudos apontam que cerca de 1/3 dos internados apresentam anemia ${ }^{11}$.

O desenvolvimento de anemia a partir de 65 anos mostra predomínio no sexo masculino, com maior acometimento após 85 anos $^{7}$. Essa distribuição entre os sexos pode ser verificada em outros estudos ${ }^{12,13}$. Nunes et al. ${ }^{12}$ mostrou que a prevalência de anemia é maior em mulheres com idade inferior a 65 anos e em homens com idade superior a 65 anos.

Entretanto, no estudo de Geisel et al. ${ }^{14}$ não se encontrou associação entre anemia e aumento da idade em pacientes hospitalizados. Nosso estudo não pôde descartar ou confirmar a associação entre idade e anemia, haja vista que apenas $14,63 \%$ do grupo amostral possuía mais de 75 anos, tornando a população mais idosa pouco representada. Isso reflete, em partes, a expectativa de vida do brasileiro de 75,5 anos, segundo dados do IBGE para 2015. Este estudo detectou maior prevalência de anemia na mediana de idade de 56,6 anos $( \pm 18,0)$, com predileção pelo sexo masculino $(55,9 \%)$ em relação ao sexo feminino $(44,1 \%)$.

A prevalência de anemia nos pacientes internados foi de $66,3 \%$ e, de forma concordante, a literatura relata grande prevalência de anemia em pacientes internados em serviços de saúde terciários, visto que está associada com diversas condições que predispõem a necessidade de internação ${ }^{1,2}$. Neste estudo o tempo médio de permanência hospitalar encontrado foi de 14,9 dias para anemia leve e 15,8 dias para anemias moderadas e graves severas $(\mathrm{p}=0,721)$. Desta forma sugere-se que a doença de base do paciente tenha maior impacto no tempo de internação do que a presença de anemia. O período estudado reflete a prevalência anual de anemias, uma vez que, não se encontram dados que indiquem um padrão de sazonalidade para a sua presença ${ }^{15}$.

Em relação ao grau de gravidade da anemia, um estudo realizado com 193 pacientes idosos internados em enfermarias de clínica médica de um hospital italiano verificou que $48 \%$ dos pacientes apresentaram anemia leve a moderada e 4,1\% anemia grave ${ }^{16,17}$. Em concordância, nosso estudo revelou uma maior prevalência de anemia moderada e leve entre os idosos, em contraste com anemia grave.

Quanto à classificação morfológica, as anemias normocíticas são descritas com maior prevalência em adultos hospitalizados ${ }^{15}$. Neste estudo, as anemias normocíticas ocorreram em 89 pacientes $(72,36 \%)$, seguida pelas microcíticas $(26,0 \%)$ e macrocíticas $(1,63 \%)$. É importante ressaltar que tal resultado pode ter sido influenciado pelo fato de que nosso hospital faz parte de uma rede de referência de tratamento de câncer, 
Oliveira MF, et al. Estudo clínico e epidemiológico das anemias em pacientes hospitalizados.

e as neoplasias geralmente são categorizadas como anemia de doença crônica e cursam mais comumente com normocitose.

Em geral, a população hospitalizada com idade superior a 65 anos preenche os critérios laboratoriais para o diagnóstico de anemia de doença crônica (ADC) ${ }^{18,19,20}$. $\mathrm{Na}$ população estudada, identificamos 99 (80,48\%) pacientes com ADC. Esta condição encontra-se associada a situações de inflamação e/ou infecções crônicas e agudas (bacteriana, fúngica e viral), além de neoplasias ${ }^{21,22}$. A ADC caracteriza-se por ser leve a moderada, normocítica e normocrômica (ocasionalmente microcítica), do tipo hipoproliferativa, com ferro sérico e saturação de transferrina reduzidos e paradoxalmente, aumento do ferro de depósito ${ }^{23}$. Segundo Ferrucci et al. ${ }^{24}$, em seu estudo com 964 pacientes hospitalizados acima de 65 anos, a principal causa de anemia foi $\operatorname{ADC}(28,1 \%)$ e o sexo masculino tem sido descrito como o de maior vulnerabilidade à anemia $^{24}$; as razões para essa maior suscetibilidade não estão esclarecidas, mas alguns autores sugerem que isso decorra de um ponto de corte de hemoglobina mais baixo para o sexo feminino ${ }^{25}$.

Pelo fato da anemia ser um sinal e não um diagnóstico etiológico, estabelecer sua causa é absolutamente necessário, a fim de implementação de terapia eficaz ${ }^{16}$. Poucos estudos têm investigado o perfil etiológico da anemia em pacientes hospitalizados ${ }^{14}$. No presente estudo o diagnóstico etiológico da anemia foi encontrado em 113 dos casos sendo que em alguns encontrou-se mais de uma 1 causa para a anemia. Quase metade dos pacientes tinham anemia secundária a neoplasia maligna, seguida das causas infeciosas. Estes achados, provavelmente refletem o perfil assistencial do hospital estudado, referência regional em assistência oncológica.

A anemia está associada a maior morbimortalidade e reinternações no primeiro ano da hospitalização. Com seis reinternações em nosso estudo, seus efeitos no desfecho da população ainda não estão bem estabelecidos. Contudo, a literatura mostra maior mortalidade associada a idosos que são re-hospitalizados, principalmente com níveis mais baixos de hemoglobina ${ }^{26}$.

À luz de Rachoin et al. ${ }^{27}$, pacientes com anemia tiveram maior risco de morte intra hospitalar e de maior tempo de internação. No entanto, estudo realizado na Austrália, concluiu que a anemia leve pode aumentar o tempo de hospitalização e foi associada a maior mortalidade entre os pacientes internados ${ }^{28}$. Em consonância com Rachoin, constatamos em nossa casuística, mortalidade superior em pacientes com anemia moderada/severa $(36,4 \%)$ em comparação com portadores de anemia leve $(18,2 \%-\mathrm{p}=0,129)^{12}$.

Esse estudo teve como limitantes a distorção da etiologia e classificação da anemia devido ao grande aporte de pacientes oncológicos e falta de significância estatística em diversas variáveis devido ao tamanho amostral. Outro viés é a contabilização do mesmo paciente mais de uma vez no estudo, quando estes apresentam internações recorrentes.

A principal contribuição deste estudo é evidenciar que a correta identificação da causa base da anemia pode permitir um tratamento resolutivo, evitando assim os efeitos deletérios da condição anêmica sobre a estado clínico do paciente. Além disto, como a anemia da doença crônica/ inflamação é a principal causa de anemia em pacientes hospitalizados, atentar aos sintomas anêmicos e não apenas atribui-los a doença de base, considerando-se terapias mais assertivas e direcionadas à esta situação clínica como suplementação de ferro e fatores estimuladores de eritropoiese, podem melhorar a qualidade de vida do paciente ${ }^{29}$.

No entanto, futuros estudos são necessários para melhor caracterizar a epidemiologia hospitalar da anemia no Brasil.

\section{CONCLUSÃO}

A anemia faz parte do espectro de condições que levam o indivíduo a ser hospitalizado, implicando em maior risco de mortalidade, especialmente nas formas mais graves, bem como maior tempo de internação. Os pacientes institucionalizados possuem maior prevalência da anemia em relação a população pelo fato de apresentarem enfermidades e disfunções orgânicas severas, tornando a anemia de doença crônica/inflamação a etiologia principal desse grupo. Mesmo evidenciando uma maior predileção pelo sexo masculino, não foi possível estabelecer uma relação significativa entre a idade e anemia, bem como o número de reinternações. Novos estudos nacionais sobre o perfil epidemiológico das anemias nos pacientes hospitalizados são necessários para corroborar as assertivas aqui encontradas.

Participação dos autores: Daniel D'Almeida Preto: Co-orientador. Juliana Rinaldis da Silva: Coleta de dados, dissertação do artigo. Revisão das normas editoriais , adaptações segundo orientação dos revisores da Revista Medicina. Mariana Franco de Oliveira: Coleta de dados, dissertação do artigo. Inserção do artigo na plataforma da revista, diagramação/formatação, revisão das normas editoriais. Adaptações segundo orientação dos revisores da Revista Medicina. Marília Franco de Oliveira: Coleta de dados, dissertação do artigo. Michael da Cruz Ferrari: Coleta de dados, dissertação do artigo. Nathalia Cavalcante Navarro: Coleta de dados, dissertação do artigo. Nathalya Fernanda Rossi: Coleta de dados, dissertação do artigo. Revisão das normas editoriais, adptação segundo orientação dos revisores da Revista Medicina.Pedro Castela Malozze: Coleta de dados, dissertação do artigo. Inserção do artigo na plataforma da revista, diagramação/formatação, revisão das normas editoriais. Adaptações segundo orientação dos revisores da Revista Medicina. Renata de Oliveira Costa: Orientadora. 


\section{REFERENCIAS}

1. Blackwell S, Hendrix PC. Common anemias: what Lies Beneath - anemias comuns clinician reviews. Medscap. 2001;11(3):53-62.

2. Nathavitharana RL, Murray JA, D'Sousa N, Sheehan T, Frampton CM, Baker BW. Anaemia is highly prevalent among unselected internal medicine inpatients and is associated with increased mortality, earlier readmission and more prolonged hospital stay: an observational retrospective cohort study. Intern Med J. 2012;42(6):683-91. doi: 10.1111/j.1445-5994.2011.02566.x.

3. Riva E, Colombo R, Moreo G, Mandelli S, Franchi C, Pasina L, Tettamanti M, Lucca U, Mannucci PM, Nobili A; REPOSI Investigators. Prognostic value of degree and types of anaemia on clinical outcomes for hospitalised older patients. Arch Gerontol Geriatr. 2017;69:21-30. doi: 10.1016/j.archger.2016.11.005.

4. Spence RK. Medical and economic impact of anemia in hospitalized patients. Am J Health Syst Pharm. 2007;64(16 Suppl 11):S3-10. doi: 10.2146\\ajhp070244.

5. Tettamanti M, Lucca U, Gandini F, Recchia A, Mosconi P, Apolone G, Nobili A, Tallone MV, Detoma P, Giacomin A, Clerico M, Tempia P, Savoia L, Fasolo G, Ponchio L, Della Porta MG, Riva E. Prevalence, incidence and types of mild anemia in the elderly: the "Health and Anemia" populationbased study. Haematologica. 2010;95(11):1849-56. doi: 10.3324/haematol.2010.023101.

6. World Health Organization - WHO. Haemoglobin concentrations for the diagnosis of anaemia and assessment of severity. Vitamin and mineral nutrition information system. Geneva; 2011 [cited 2017 Jan]. Available from: http://www. who.int/vmnis/indicators/haemoglobin.pdf.

7. Guralnik JM, Eisenstaedt RS, Ferrucci L, Klein HG, Woodman RC. Prevalence of anemia in persons 65 years and older in the United States: evidence for a high rate of unexplained anemia. Blood. 2004;104(8):2263-8. doi: 10.1182/blood-2004-05-1812.

8. Beutler E, Waalen J. The definition of anemia: what is the lower limit of normal of the blood hemoglobin concentration? Blood. 2006;107(5):1747-50. doi: 10.1182/ blood-2005-07-3046.

9. Patel KV. Epidemiology of anemia in older adults. Semin Hematol. 2008;45(4):210-17. doi: $10.1053 / \mathrm{j}$. seminhematol.2008.06.006.

10. Patel KV, Harris TB, Faulhaber M, Angleman SB, Connelly S, Bauer DC, Kuller LH, Newman AB, Guralnik JM. Racial variation in the relationship of anemia with mortality and mobility disability among older adults. Blood. 2007;109(11):4663-70. doi: 10.1182/blood-2006-10-055384.

11. Means RT Jr, Krantz SB. Progress in understanding the pathogenesis of the anemia of chronic disease. Blood. 1992;80(7):1639-47.

12. Robalo Nunes A, Fonseca C, Marques F, Belo A, Brilhante $\mathrm{D}$, Cortez J. Prevalence of anemia and iron deficiency in older Portuguese adults: an EMPIRE substudy. Geriatr Gerontol Int. 2017;17(11):1814-1822.. doi: 10.1111/ggi.12966.

13. Skjelbakken T, Langbakk B, Dahl IM, Løchen ML; Tromsø Study. Haemoglobin and anaemia in a gender perspective: the Tromsø Study. Eur J Haematol. 2005;74(5):381-8. doi: 10.1111/j.1600-0609.2004.00392.x.

14. Geisel T, Martin J, Schulze B, Schaefer R, Bach M, Virgin G, Stein J. An etiologic profile of anemia in 405 geriatric patients. Anemia. 2014;2014:932486. doi: 10.1155/2014/932486.
15. Corrêa M, Baldessar MZ, Fissmer LEW, Fissmer JFW. Prevalência das anemias em pacientes hospitalizados. ACM Arq Catarin Med. 2004;33(1):36-41. Disponível em: http:// www.acm.org.br/revista/pdf/artigos/171.pdf.

16. Muñoz RL, Mendonça MS, Silva ELVF, Araújo DU. Prevalência de anemia em idosos internados em enfermarias clínicas do Hospital Universitário da UFPB. Anais CIEH. 2015;2(1): Disponível em: https://editorarealize.com.br/ revistas/cieh/trabalhos/TRABALHO_EV040_MD2 SA3 ID1733_17072015140652.pdf.

17. Migone De Amicis M, Poggiali E, Motta I, Minonzio F, Fabio G, Hu C, Cappellini MD. Anemia in elderly hospitalized patients: prevalence and clinical impact. Intern Emerg Med. 2015;10(5):581-6. doi: 10.1007/s11739-015-1197-5.

18. Joosten E, Pelemans W, Hiele M, Noyen J, Verhaeghe R, Boogaerts MA. Prevalence and causes of anaemia in a geriatric hospitalized population. Gerontology. 1992;38(12):111-7. doi: 10.1159/000213315.

19. Alves RA, Miszputen SJ, Figueiredo MS. Anemia in inflammatory bowel disease: prevalence, differential diagnosis and association with clinical and laboratory variables. Sao Paulo Med J. 2014;132(3):140-6.

20. Cash JM, Sears DA. The anemia of chronic disease: spectrum of associated diseases in a series of unselected hospitalized patients. Am J Med. 1989;87(6):638-44. https://doi. org/10.1016/S0002-9343(89)80396-1.

21. Means RT Jr. Advances in the anemia of chronic disease. Int J Hematol. 1999;70:7-12.

22. Means RT Jr, Krantz SB. Progress in understanding the pathogenesis of the anemia of chronic disease. Blood. 1992;80(7):1639-47.

23. Price EA, Mehra R, Holmes TH, Schrier SL. Anemia in older persons: etiology and evaluation. Blood Cells Mol Dis. 2011;46(2):159-65. doi: 10.1016/j.bcmd.2010.11.004.

24. Ferrucci L, Guralnik JM, Woodman RC, Bandinelli S, Lauretani F, Corsi AM, et al. Proinflammatory state and circulating erythropoietin in persons with and without anemia. Am J Med. 2005;118(11):1288. doi: 10.1016/j. amjmed.2005.06.039.

25. Tomiya MTO, Pinho CPS, Cabral PC. Anemia e fatores associados: um estudo em idosos hospitalizados. Rev Bras Nutr Clin. 2014;29(4):296-303. Disponível em: http://www. braspen.com.br/home/wp-content/uploads/2016/11/04Anemia-e-fatores-associados.pdf.

26. Culleton BF, Manns BJ, Zhang J, Tonelli M, Klarenbach S, Hemmelgarn BR. Impact of anemia on hospitalization and mortality in older adults. Blood. 2006;107(10):3841-6. doi: 10.1182/blood-2005-10-4308.

27. Rachoin JS, Cerceo E, Milcarek B, Hunter K, Gerber DR. Prevalence and impact of anemia in hospitalized patients. South Med J. 2013;106(3):202-6. doi: 10.1097/ SMJ.0b013e318287fcd9.

28. Nseir W, Artul S, Nasrallah N, Mograbi J, Mahamid M. Hospitalization and 1-year all-cause mortality in type 2 diabetic patients with chronic kidney disease at stages 1 and 2: effect of mild anemia. J Diabetes. 2016;8(4):502-7. doi: 10.1111/1753-0407.12318.

29. Weiss G, Ganz T, Goodnough LT. Anemia of inflammation. Blood. 2019;133(1):40-50. doi: 10.1182/ blood-2018-06-856500.

Recebido: 25.04.18

Aceito: 19.02 .19 